Short Report

\title{
Validation of rapid tests to detect urinary tract infection
}

\author{
K Manoharan ${ }^{1}$, T Kumanan ${ }^{2}$, K Murugananthan ${ }^{3}$ \\ Sri Lankan Journal of Infectious Diseases 2016 Vol.6 (2):126-129 \\ DOI: http://dx.doi.org/10.4038/sljid.v6i2.8113
}

\begin{abstract}
There are several rapid dip stick tests currently in use to detect urinary tract infection (UTI). This study was carried out to validate three different dip sticks that are widely used with urine culture which is the gold standard to diagnose UTI. The findings of the current study suggest that the sensitivity, specificity and predictive value of the three dipsticks are not statistically different from each other and are comparable to sedimentary microscopy. These these three dipsticks can therefore be used instead of urinary sedimentation method. However low positive predictive value (PPV) of all three dipsticks is a major limitation. Further studies with large sample size are needed to evaluate their value in clinical practice.
\end{abstract}

Key words: $\quad$ Urinary tract infection, Leukocyte esterase, Nitrite, Sediment microscopy, Urine analysis

\section{Introduction}

Urinary tract infection (UTI) is a major economic burden with up to $30 \%$ of women having recurrent UTI in their life time. ${ }^{1}$ UTI in men are less common but increases after the age of 50 years. Although urinary culture is the gold standard for the diagnosis of UTI, urine sediment microscopy and several different urine dipstick rapid tests have become popular. ${ }^{2}$ While being rapid and relatively cheap, standardization of these tests have become problematic. This study was carried out to validate three different commercially available dipsticks used in Sri Lankan laboratories with sediment microscopy and urine culture.

In urine sediment microscopy, the presence of $>5$ pus cells per high power field (HPF) is suggestive of UTI. ${ }^{3}$ Leukocyte esterase (LE) and nitrite (NT) can also be used as markers for screening for UTI by urine dipsticks. ${ }^{4}$ LE is an enzyme produced by granulocytes, mostly neutrophils. It is an indicator of pyuria as it detects both the lysed and intact granulocytes. LE test may be falsely negative if there are less than 100 neutrophils per $10 \mathrm{HPF}^{5}$ False positive results may be due to contamination with squamous epithelial cells. NT is positive with nitrite reducing bacteria which causes $\mathrm{UTI}^{6,7,8}$ as these organisms convert dietary nitrates present in the

${ }^{1}$ Unit of Allied Health Sciences, faculty of Medicine, University of Jaffna

${ }^{2}$ Department of Medicine, Faculty of Medicine, University of Jaffna

${ }^{3}$ Division of Microbiology, Department of Pathology, Faculty of Medicine, University of Jaffna

Address for correspondence: Dr Kalamathy Murugananthan, Division of Microbiology, Department of Pathology, Faculty of Medicine, University of Jaffna Telephone : +94 212222073 / +94 777742349

email : kalamathy6@gmail.com 
urine to nitrites. False positive results commonly occur due to the poor collection and storage. False negative results on the other hand, may be due to low $\mathrm{pH}(<6)$ and/or a high content of ascorbic acid and urobilinogen., ${ }^{4,9}$

\section{Methodology}

This laboratory based cross sectional study included 116 mid-stream urine samples from adults and clean-catch urine specimens from infants suspected of having UTI. All the urine specimens were cultured using a $1 \mu \mathrm{L}$ calibrated loop. An aliquot of the urine specimen was streaked on a cysteine lactose electrolyte deficient agar (CLED) plate using aseptic techniques. The plates were incubated at $37{ }^{\circ} \mathrm{C}$ for 24 hours. Isolated colonies were counted and reported as the number of bacteria per milliliter of urine. Further organism identification was done using Gram staining, motility test and standard biochemical tests.

Urine dipsticks from three different manufactures (A- UroColorTM 10, Standard Diagnostic Inc., Korea; B- URS-10, Teco Diagnostics, USA; C -Urine 10, Cypress diagnostics, Belgium) were used according to the manufacturer's instructions.

Five milliliters from each urine specimen was centrifuged at $5000 \mathrm{~g}$ for 5 minutes and the sediment examined under the microscope for the number of pus cells present. Pyuria was defined as $>$ than five white blood cells per HPF.

The results from the LE and NT of all three dipsticks and sediment microscopy detection were compared with urine culture as the gold standard. The test accuracy indices were determined by calculating the sensitivity, specificity, PPV and NPV using standard formulae. ${ }^{10}$

\section{Results and Discussion}

Of the 116 urine specimens cultured, organisms were isolated in 32 which were positive with a bacterial count of $>10^{4} / \mathrm{ml}$. The vast majority of the positive samples had E. coli $(41 \%)$ followed by Pseudomonas spp (22\%), Proteus spp (13\%), Citrobacter spp (9\%), Klebsiella spp (6\%), Streptococcus spp (3\%) and Candida albicans (3\%).

Table 1 shows the specificity of NT in urine dipsticks A, B and C to be $85.8 \%, 85.7 \%$ and $85.4 \%$ respectively. However, the sensitivity of NT in all three urine dipsticks was found to be low at $45.1 \%, 46.8 \%$ and 43.7 respectively. Our observations are supported by previous studies in which the NT test has been found to have high specificity and low sensitivity (below 30\%) ${ }^{11,12}$, while the study by Kacmaz and colleagues ${ }^{13}$ found the sensitivity of NT to be relatively high $(60 \%)$.

In the current study, the LE tests of all three urine dipsticks also showed a higher sensitivity of $81.2 \%, 81.2 \%$ and $84.3 \%$ whereas the specificity was comparatively low at $60.7 \%, 60.7 \%$ and $60.7 \%$ respectively. 
Table 1 : Comparison of sediment microscopy and dipstick tests with urine culture

\begin{tabular}{|c|c|c|c|c|c|c|c|}
\hline \multirow[b]{2}{*}{ 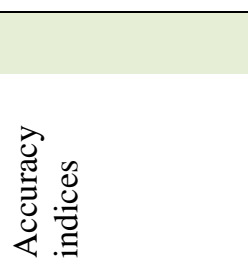 } & \multirow[b]{2}{*}{ 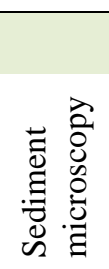 } & \multicolumn{2}{|c|}{ Strip A } & \multicolumn{2}{|c|}{ Strip B } & \multicolumn{2}{|c|}{ Strip C } \\
\hline & & 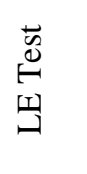 & $\begin{array}{l}\stackrel{\vec{s}}{\leftrightarrow} \\
\stackrel{5}{\mathrm{z}}\end{array}$ & 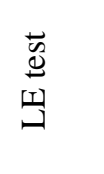 & 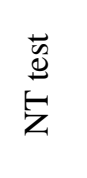 & 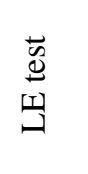 & 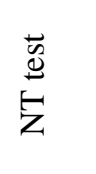 \\
\hline Sensitivity (\%) & 81.2 & 81.2 & 45.1 & 81.2 & 46.8 & 84.3 & 43.7 \\
\hline & 61.9 & 60.7 & 85.8 & 60.7 & 85.7 & 60.7 & 80.4 \\
\hline & 44.8 & 44.8 & 53.8 & 44.0 & 55.5 & 45.0 & 58.3 \\
\hline NPV (\%) & 89.6 & 89.7 & 81.1 & 89.4 & 80.8 & 91.0 & 80.4 \\
\hline
\end{tabular}

$\mathrm{PPV}=$ positive predictive value $\mathrm{NPV}=$ negative predictive value

Using sediment microscopy, pyuria was detected in 58 specimens. However, only 26 were culture positive and the sensitivity and the specificity of sediment microscopy was similar to LE test of all three dipsticks. Although Shaw et al(1998) ${ }^{14}$ reported that the microscopic pyuria as assessed by sediment microscopy was sensitive in detecting UTI, it was prone to more false positive results than the urine dipstick test.

In the current study, accuracy indices were calculated using urine culture as the gold standard. Significant differences $(p>0.05)$ in NT and LE were not detected in any of the indices between the three dipsticks. Similarly, no difference was found between sediment microscopy and LE.. The low PPV (ranging from 44-58\%) of LE and NT detection and low specificity (60.7\%) of the LE test with all 3 dipsticks suggests a high possibility of false negatives and false positives using these rapid tests. The use of such dipsticks cannot therefore replace urine culture in the diagnosis of urinary tract infection. However, further studies with large sample size would be useful to determine the indications for use of these tests.

\section{Conclusion}

In conclusion, although the three urine dipsticks are not significantly different from each other in either sensitivity or specificity of NT or LE detection, the possibility of both false negative and false positive results should be considered when used in clinical practice. Further studies are indicated to determine their role in the diagnosis of UTI, particularly in clinical environments where culture facilities are unavailable.

\section{Acknowledgements}

We would like to acknowledge the contribution of Professor Natkunam Ketheesan from the Australian Institute of Tropical Health and Medicine, James Cook University for the critical review of this manuscript. 


\section{References}

1. Giesen LGM, Cousins G, Dimitrov BD, et al. Predicting acute uncomplicated urinary tract infection in women: a systematic review of the diagnostic accuracy of symptoms and signs. BMC Family Practice 2010; 11:78 doi : http://dx.doi.org/10.1186/1471-2296-11-78

2. Memişoğullar R, Yüksel H, Yıldırım HA, Yavuz Ö. Performance characteristics of dipstick and microscopic urinalysis for diagnosis of urinary tract infection Eur J Gen Med 2010; 7(2):174-178 No doi

3. Oladeinde $\mathrm{BH}$, Omoregie R, Oladeinde OB. Asymptomatic urinary tract infection among pregnant women receiving ante-natal care in a traditional birth home in Benin city, Nigeria. Ethiopian Journal of Health Sciences. 2015; 1:25(1):3-8. www.ncbi.nlm.nih.gov/pubmed/25733779

4. Najeeb S, Munir T, Rehman S, et al. Comparison of urine dipstick test with conventional urine culture in diagnosis of urinary tract infection. Journal of the College of Physicians and Surgeons-Pakistan 2015; 25(2):108-10. www.ncbi.nlm.nih.gov/pubmed/25703753

5. Coad S, Friedman B, Geoffrion R. Understanding urinalysis Expert Rev of Obstet Gynecol. 2012; 7(3):269-279 doi : http://dx.doi.org/10.1586/eog.12.21

6. Robertson AW, Duff P. The nitrite and leukocyte esterase tests for the evaluation of asymptomatic bacteriuria in obstetric patients. Obstet Gynecol 1988; 71(6 Pt 1):878-81. www.ncbi.nlm.nih.gov/pubmed/3285267

7. Panesar k. Urinalysis: A Guide for Pharmacists. 2009 Available at: http://www.uspharmacist.com/continuing_education/ceviewtest/lessonid/106254/

8. Wilson ML, Gaido L. Laboratory diagnosis of urinary tract infections in adult patients. Clinical Infectious Diseases. 2004; 38(8):1150-1158.

doi: http://dx.doi.org/10.1086/383029

9. Mambatta, Anith Kumar, et al. Reliability of dipstick assay in predicting urinary tract infection. Journal of Family Medicine and Primary Care 2015; 4(2):265-268 doi: http://dx.doi.org/10.4103/2249-4863.154672

10. Sivagnanasundram.C 2003 Learning Research 2nd Edition ASBN 955-96654-05 BoascoArton Press: 107-11

11. Juthani-Mehta M, Drickamer MA, Towle V, et al. Role of dipstick testing in the evaluation of urinary tract infection in nursing home residents. Infect Control Hosp Epidemiology 2007; 28(7):889-91 doi : http://dx.doi.org/10.1086/518752

12. Eidelman. Y, Raveh. D, Yinnon. A. M., et al Reagent strip diagnosis of UTI in high risk population. Am J Emergency Med 2002; 20(2):112-3 doi : http://dx.doi.org/10.1053/ajem.2002.31145

13. Kacmaz.B., Cakir O, Aksoy.A, Biri A Evaluation of rapid urine screening tests to detect asymptomatic bacteriuria in pregnancy. Jpn J Infect Dis 2006; 59:261-63 No doi

14. Shaw KN, McGowan KL, Gorelick MH, Schwartz JS Screening for urinary tract infection in infants in the emergency department: which test is best? Pediatrics 1998; 101(6):E1 www.ncbi.nlm.nih.gov/pubmed/9606243 